\title{
Aracna: An Open-Source Quadruped Platform for Evolutionary Robotics
}

\author{
Sara Lohmann*, Jason Yosinski*, Eric Gold, Jeff Clune, Jeremy Blum and Hod Lipson \\ Cornell University, 239 Upson Hall, Ithaca, NY 14853 \\ sml253@cornell.edu, yosinskidcs.cornell.edu
}

\begin{abstract}
We describe a new, quadruped robot platform, Aracna, which requires non-intuitive motor commands in order to locomote and thus provides an interesting challenge for gait learning algorithms, such as those frequently developed in the Evolutionary Computation and Artificial Life communities. Aracna is an open-source hardware project composed of off-the-shelf and 3D-printed parts, enabling other research teams to modify its design according to their scientific needs. Aracna was designed to overcome the shortcomings of a previous quadruped robot platform, whose legs were so heavy that the motors could not reliably execute the commands sent to them. We avoid this problem by locating all motors in the body core instead of on the legs and through a design which enables the servos to have a greater mechanical advantage. Specifically, each of the four legs has two joints controlled by separate four-bar linkage mechanisms that drive the pitch of the hip joint and knee joint. This novel design causes unconventional kinematics, creating an opportunity for gaitlearning algorithms, which excel in counter-intuitive design spaces where human engineers tend to underperform. Because it is low-cost, flexible, kinematically interesting, and and improvement over a previous design, Aracna provides a useful new hardware platform for testing algorithms that automatically generate robotic behaviors.
\end{abstract}

\section{Introduction}

There is a long history in the Artificial Life and Evolutionary Robotics community of automatically generating behaviors for robots (Nolfi and Floreano, 2000; Pfeifer et al., 2007; Sims, 1994; Hornby et al., 2005; Lipson and Pollack, 2000). Much work has focused on evolving gaits for legged robots (Clune et al., 2009, 2011; Hornby et al., 2005, 2003; Kodjabachian and Meyer, 1998; Koos et al., 2011; Bongard et al., 2006; Yosinski et al., 2011; Gallagher et al., 1996). While some of this previous work involved evolution directly on a physical robot (Yosinski et al., 2011; Zykov et al., 2004), more often a gait was evolved in simulation and then transferred to the physical robot (Lipson et al., 2006; Koos et al., 2011; Hornby et al., 2005; Bongard et al., 2006). Many of these studies report that evolutionary algorithms

\footnotetext{
${ }^{*}$ Authors contributed equally to this work.
}

produced gaits that outperformed those designed by a human engineer (Yosinski et al., 2011; Hornby et al., 2005), which is not surprising given that evolutionary algorithms routinely create solutions that are superior to manually created solutions (Koza, 2003).

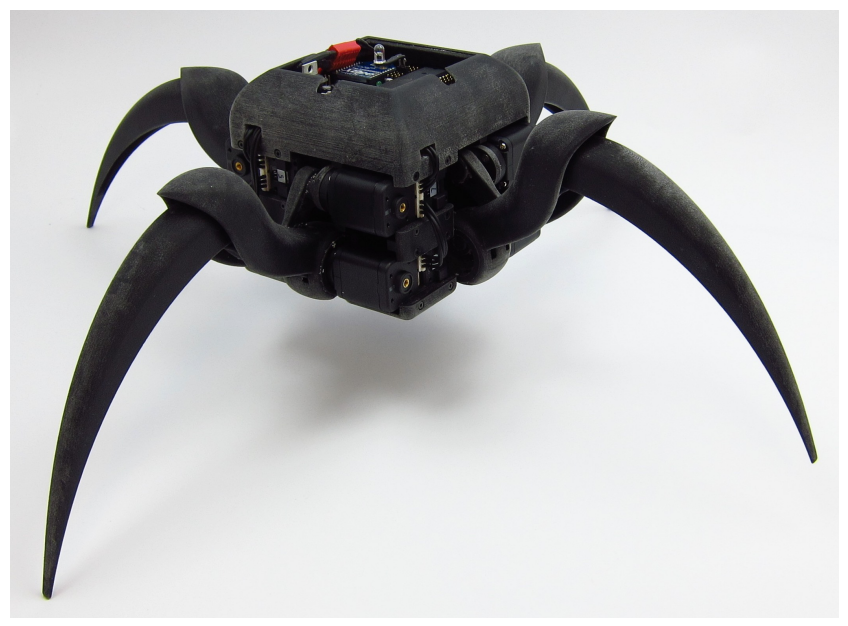

Figure 1: Aracna: an open-source 3D printed quadruped robot platform, here printed in a black rapid prototyping polymer. STL files for 3D printing the robot and drivers for the Arbotix board and servos are publicly available at http://creativemachines.cornell.edu/aracna.

The results just mentioned suggest that evolutionary algorithms are a promising approach for generating gaits and other behaviors for physical robots. Despite this promise, the field remains small, partly because robots are expensive, and they are difficult to modify. Access to cheap, customizable robots could increase the number of researchers able to participate in the field. Moreover, in nearly all of the papers mentioned previously, the robots were custom-made, preventing teams at other universities from reproducing the results of other groups and or testing new algorithms on a robotic platform used in a previous study. That, in turn, slows the progress of science because it is difficult to interpret whether the variance in results between different studies 
was due to the algorithms used or the robotic platform those algorithms were tested on.

Some robot platforms are emerging, but they tend to be wheeled robots without complex kinematics, such as the ePuck (Mondada et al., 2009). Wheeled robots are interesting testbeds for many robotic behaviors, but they do not allow gait evolution and are unable to traverse rugged terrains. Legged robotic platforms exist, but they tend to be extremely expensive, such as the Aldebaran Nao, which costs more than $\$ 10,000$ USD. Another drawback to these commercial platforms is that it is hard, if not impossible, to modify the hardware design because they are not open-source hardware projects, and do not take advantage of off-the-shelf components and 3D printing, meaning that complex manufacturing tools are required to manufacture newly designed parts.

In this paper we address these needs by introducing Aracna, a low-cost, open-source, easily customizable robot platform with non-intuitive walking kinematics (Figure 1). Aracna is the third quadruped robot developed for evolutionary learning algorithms by the Creative Machines Lab at Cornell University (Bongard et al., 2006; Yosinski et al., 2011). Like the most recent of the two previous designs, called QuadraTot (Yosinski et al., 2011), the body of Aracna is $3 \mathrm{D}$ printed and the STL files are available online, meaning that other researchers can easily customize the body's design. As in the both previous designs, each leg has a hip and knee joint controlled by two actuators. The original Creative Machines Lab quadruped robot favored starfish-like movements (Bongard et al., 2006). The second quadruped robot - QuadraTot - developed spider-like movements, but was found to be limited by its weight and lack of mechanical advantage, such that the motors would overheat and timeout when trying to execute many commands (Yosinski et al., 2011; Glette et al., 2012). We designed Aracna to be able to produce fast, spider-like movements, yet be lightweight enough that the motors would not overheat. We also designed Aracna to be inexpensive: as described below, its overall price is under $\$ 1,400$ USD. In the following sections we describe the Aracna platform in more detail.

\section{Overall Hardware Design}

The hardware of Aracna was designed to improve upon the previous Creative Machines Lab quadruped robots (Bongard et al., 2006; Yosinski et al., 2011), while still qualitatively resembling those robots. Aracna is similar in that it has a body and four legs, with each leg having two joints that can pitch forward and back like knees (Figure 2 and 3).

One change was to constrain the movement of the joints toward the goal of creating faster, spider-like movements. To prevent starfish-like movements and instead encourage a walking gait with the robot body permanently off the ground, the legs were constrained such that they cannot straighten out and the knee cannot hyperextend.

Another change was to reduce the both the overall weight of the robot and the weight of each leg. Two previous studies that used the QuadraTot robot report that the motors quickly wore out and could not reliably execute the commands sent to them, likely because of both the overall weight of the QuadraTot and the fact that housing servos on the legs made them heavy (Bongard et al., 2006; Yosinski et al., 2011). The weight of the robot's core was reduced in a number of ways.

Initially, we eliminated the QuadraTot's fit-PC, an onboard Linux computer weighing $370 \mathrm{~g}$, and replaced it with an on-board ArbotiX microcontroller that weights only 47g. Wireless communication between the external control computer and the ArbotiX microcontroller occurs over wireless XBee.

A second means of eliminating weight involved switching to a lighter battery. The QuadraTot had two $12 \mathrm{~V}$ lithiumion battery packs that weighted $140 \mathrm{~g}$ each for a total of 280g. Aracna has a single lithium-polymer 11.1V battery that weighs $122 \mathrm{~g}$. As with the QuadraTot, Aracna can also run tethered to power, if desired, to avoid the need to run from battery power. This may be helpful for extended experiments.

A major modification, targeted at reducing the weight of legs, was the use of two four-bar mechanisms to drive the joints in each leg. This mechanism causes the controlled joint to move at a fraction of the output angle of the actuator, giving the motor a relatively larger mechanical advantage over the position of each leg. Figure 3 shows the crankrocker system, where the input crank is actuated by a servo and the rocker is the leg. This configuration allows the servo motors to be contained in the robot core, reducing both the inertia and mass of each leg. The weight of an Aracna leg is $105 \mathrm{~g}$ compared to the $217 \mathrm{~g}$ for a QuadraTot leg.

Combined, these changes to minimize weight led to a 31.4 percent reduction in weight of the robot. The QuadraTot weighs $1.88 \mathrm{~kg}$ whereas Aracna weighs $1.29 \mathrm{~kg}$.

A final change was to upgrade the power of the servo motors in order to increase the ability of the robot to strike whichever configurations are specified by the learning algorithms. Specifically, we upgraded from Dynamixel AX 12+ motors to AX-18A motors, which have a higher stall torque $(1.8 \mathrm{Nm}$ vs. $1.5 \mathrm{Nm}$ at $12 \mathrm{~V})$, a higher stall current $(2.2 \mathrm{~A}$ vs $1.5 \mathrm{~A}$ ), and a higher no-load speed (97 vs. 59 RPM).

\section{D Printed Body}

The body of Arcana takes advantage of 3D printing technology, also known as additive manufacturing, which generates physical objects from digital designs by building them up layer by layer (Gibson et al., 2009; Lipson and Kurman, 2010). The use of $3 D$ printing means that other Aracna users can easily make copies of Aracna, either by having access to a $3 \mathrm{D}$ printer or via online $3 \mathrm{D}$ printing services such as Shapeways, Sculpteo, or other online vendors. Either option requires the 3D design files in the stereolithography (STL) format, which are published in the online support material 


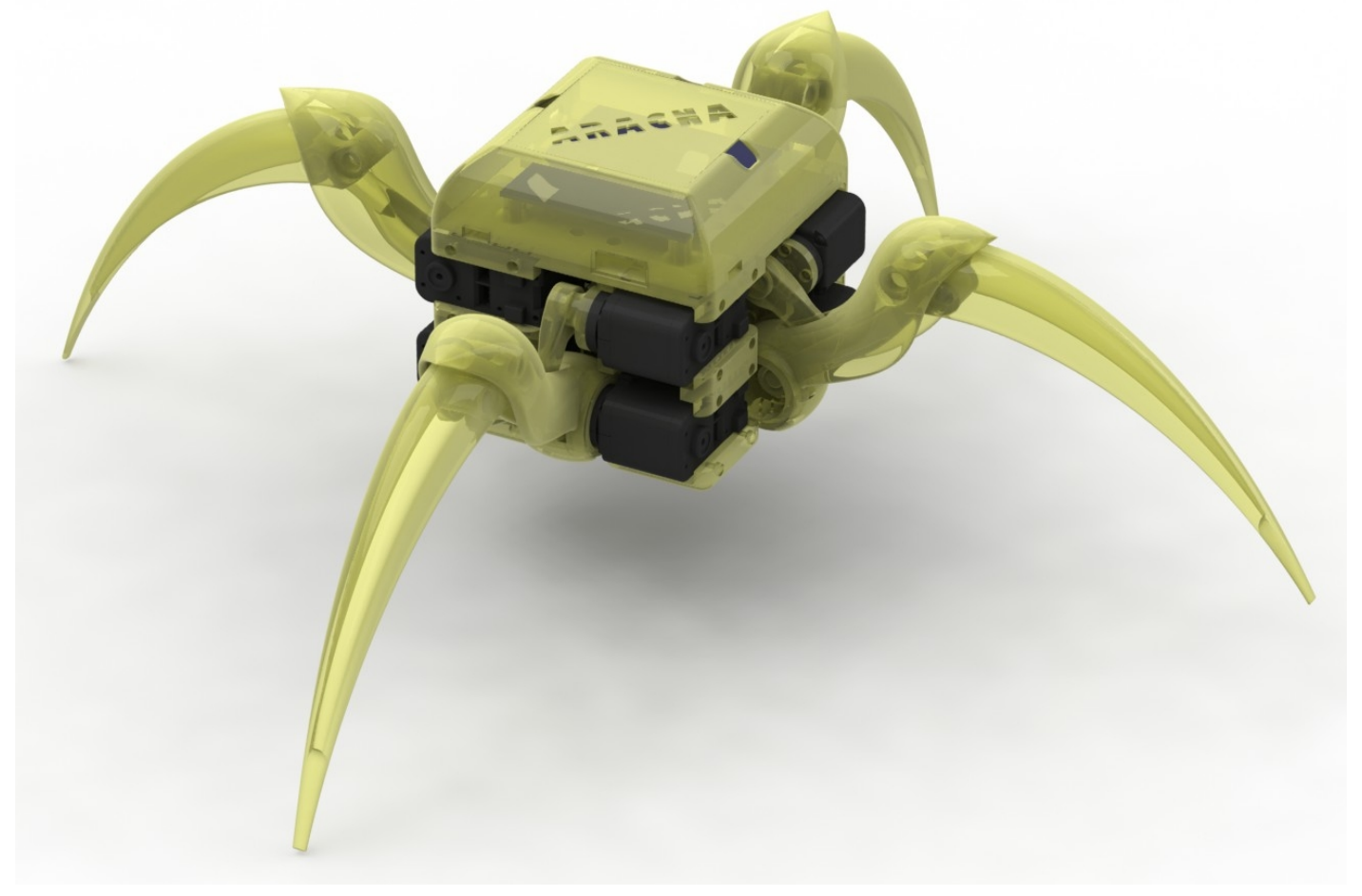

Figure 2: A rendered CAD model of Aracna. Note the lack of heavy servos on the legs themselves, which are instead controlled via four-bar linkages by servos in the robot's core.

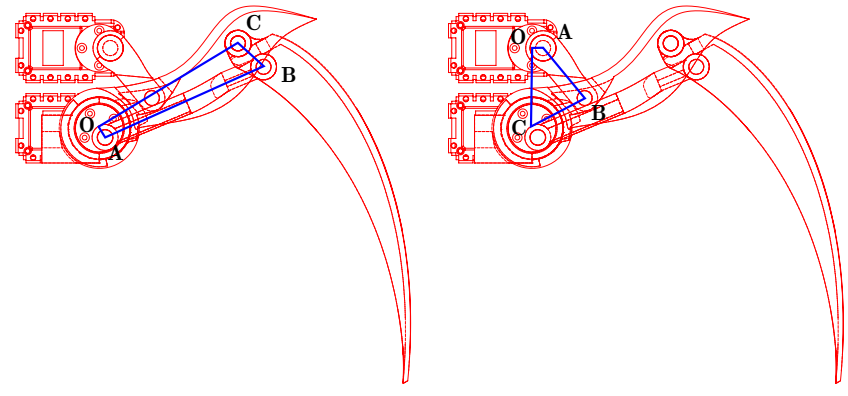

Figure 3: Crank-rocker four-bar linkage controlling flexion/extension of the knee and hip joints. In both cases above, the input crank (link OA) is actuated by a servo, the rocker is the leg (link CB), and the fixed link is OC. for this paper (Aracna, 2012). Moreover, to catalyze innovation in this open-source hardware project, we are also providing the source files for the SolidWorks computer-aideddesign program, to enable others to modify the design. It is thus possible for future Aracna users to improve or alter the design and quickly obtain a physical instantiation of the design. Importantly, the use of 3D printing eliminates the need to know how to machine parts, allowing many more researchers to participate in using physical robot morphologies that they design themselves. These ideas are in line with a broader trend toward enabling non-technical users to design and manufacture physical objects (Clune and Lipson, 2011; Clune et al., 2013; Lipson and Kurman, 2010).

An initial version of Aracna was designed to be printed in one piece (Figure 5). However, if one part of the robot became damaged, an entire new robot had to be reprinted, which took over 26 hours and costs roughly \$355 USD on an Objet Connex500 printer. To make repairing the robot easier, cheaper, and quicker, Aracna was redesigned to be modular. It consists of 15 pieces-four legs and the core-that can be separately 3D printed (Figure 6). Printing a leg takes 3.3 hours and costs roughly \$64 USD. Printing the core takes 3 hours and costs \$101 USD. All 15 Aracna pieces can still be printed as one print job, with an overall time of approximately 10 hours and cost of $\$ 308$. These figures are based 

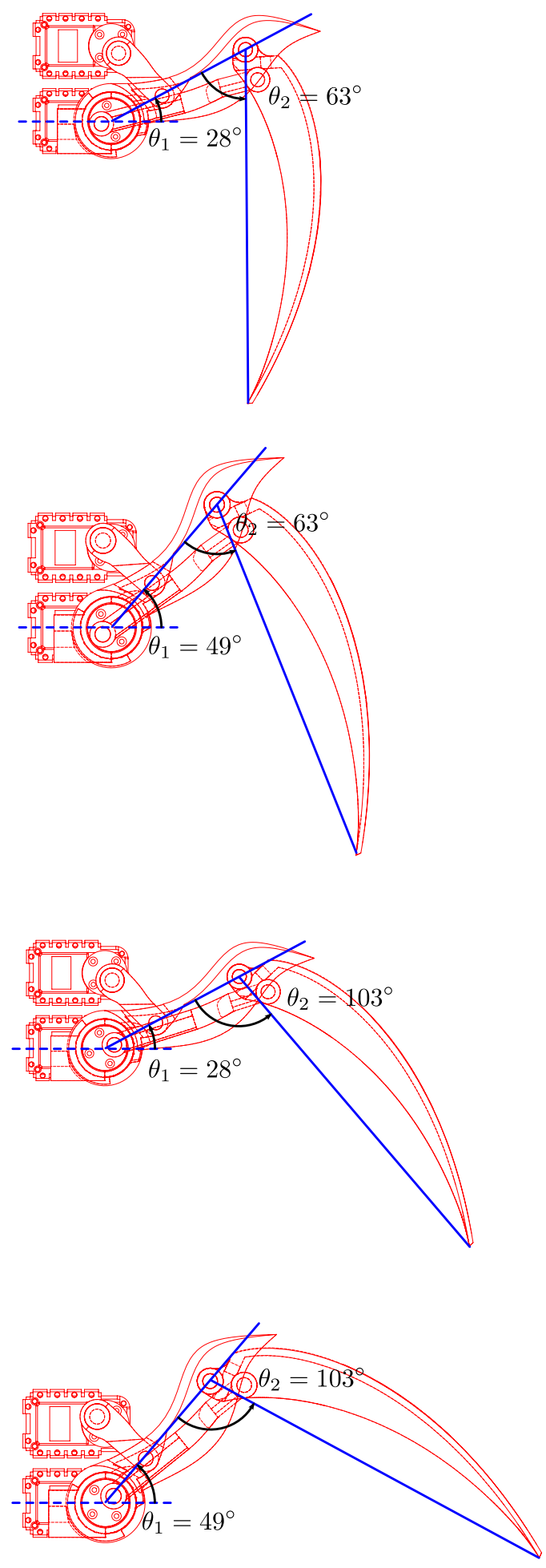

Figure 4: The range of motion of the hip and knee joints in each leg of Aracna. The hip joint rotates by $21.3^{\circ}$ and the knee joint by $40.2^{\circ}$. on Aracna's use of approximately $967 \mathrm{~g}$ of model material and $746 \mathrm{~g}$ of support material, and current material costs of $4.5 \mathrm{~g}$ per USD and $8 \mathrm{~g}$ per USD for rigid and support material, respectively. This cost estimate is variable depending on the type of material used. The print times are estimates calculated by the Objet's software and are meant to be used as a relative comparison of print times. Table 1 outlines the total estimated cost of Aracna, which is just under $\$ 1,400$, including its off-the-shelf electronic components.

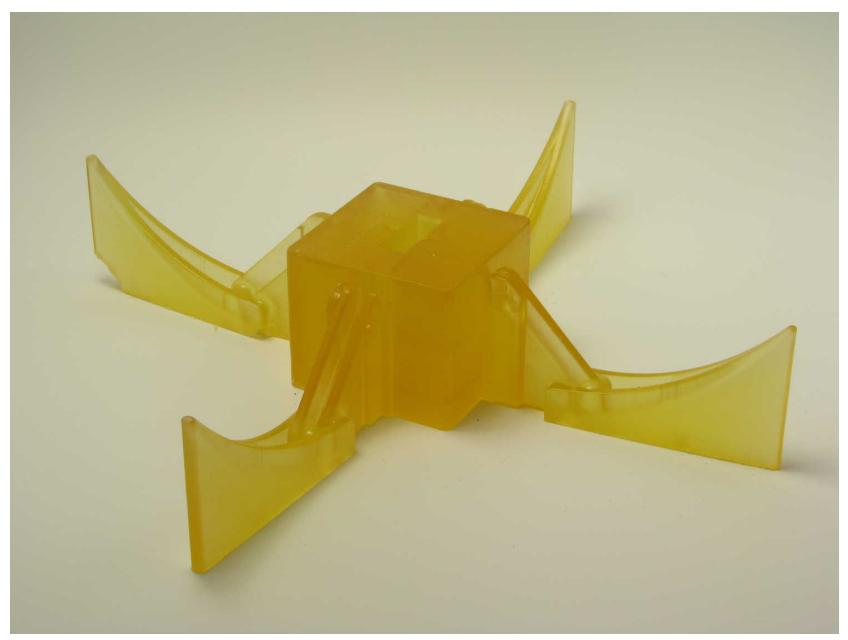

Figure 5: A draft version of Aracna that was printed in one piece. This monolithic design proved expensive to maintain if part of the robot was damaged, and was replaced in a later version with a modular design. This figure shows the printed body with support material still present.

\begin{tabular}{|c|c|}
\hline Part & Cost \\
\hline \hline 3D Print Materials & $\$ 308$ \\
ArbotiX Robocontroller Kit & $\$ 189$ \\
Dynamixel AX-18A Robot Actuator (x8) & $\$ 721$ \\
3S 11.1V 2000mAh Pro Lite LiPo Battery & $\$ 73$ \\
LiPo Battery Balance Charger Kit & $\$ 70$ \\
Cables, Connectors, Misc & $\$ 28$ \\
\hline \hline Total & $\mathbf{\$ 1 3 8 9}$ \\
\hline
\end{tabular}

Table 1: Estimated total cost. The cost of components and printing material reflect market prices from March 2012. A complete parts list is on our website (Aracna, 2012).

\section{Control}

In addition to reducing the weight of the legs, the fourbar mechanisms also satisfied the design goal of making a robot that had non-traditional movements. Unusual kinematics make for a more effective algorithmic test platform, since gait learning algorithms are most helpful in domains 


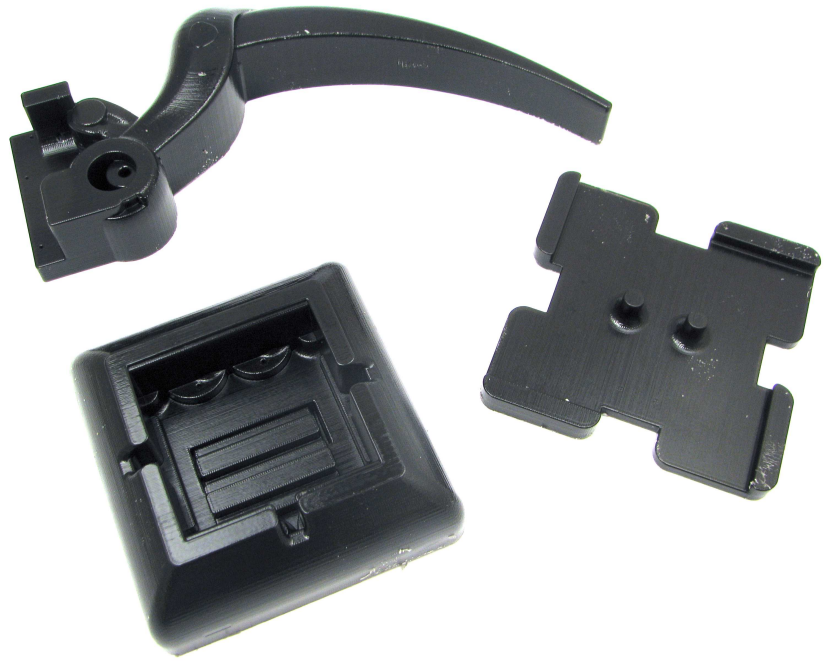

Figure 6: A final version of Aracna printed in multiple pieces. The body is printed as two pieces, with 9 smaller parts within the top piece to reduce support material and print time. These parts can be printed individually if replacements are necessary. The body consists of a total of 11 parts. This image shows a set of 12 printed parts (the complete body and a single leg) with support material still present.

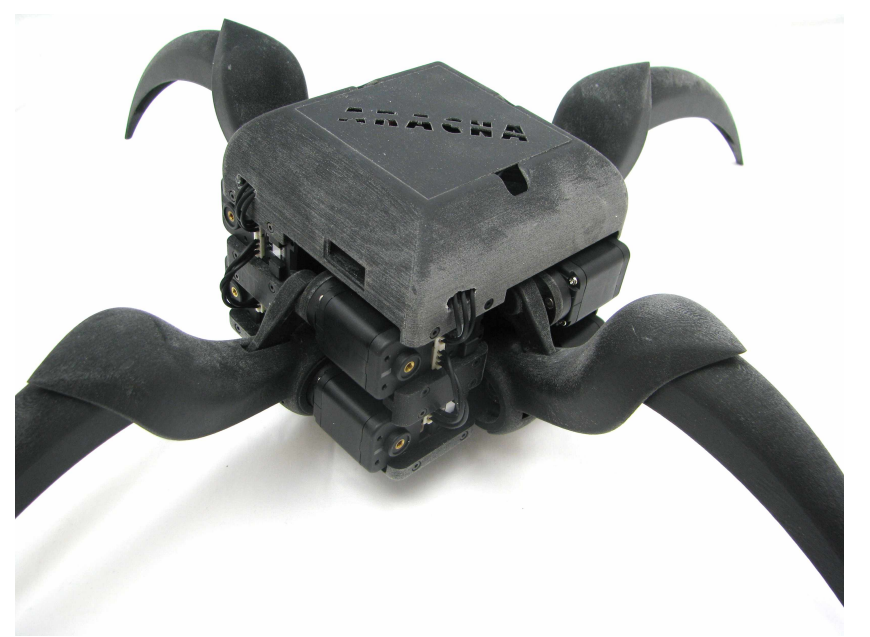

Figure 7: Aracna with optional top cover. that humans find hard to program solutions for. The reason Aracna's kinematics are counter-intuitive is because there is a nonlinear mapping between each servo's output and the movement of the joint controlled by that servo.

The range of motion for the hip joint is $21.3^{\circ}$ and that of the knee joint is $40.2^{\circ}$. These ranges are realized over a servo motion of $184^{\circ}$ and $192^{\circ}$ for the hip and knee joints, respectively. These amounts are notably larger than the corresponding joint motions, which produces the desired mechanical advantage.

There are two paradigms for encoding movements for Aracna, increasing its flexibility as a testing platform. The first method is to specify explicitly a sequence of positions over time for all eight servos. The second method is to set the speed of each servo. This method is possible because with the four bar mechanism a servo constantly rotating in one direction will move the joint back and forth between its minimum and maximum opening angle. This latter method provides a much smaller search space and would encourage regular gaits, which have been shown to be beneficial when evolving gaits for legged robots (Clune et al., 2011; Hornby et al., 2005).

\section{Software}

The software, which is also open-source and freely available (Aracna, 2012), is written in Python and based on the code developed for the QuadraTot platform (Yosinski et al., 2011). The software translates a series of requested joint angles from the learning algorithm into servo movements. Additionally, it returns information to the learning algorithm, such as the distance traveled or the specific trajectory the robot took, so the learning algorithm can assess the quality of the gait. To provide this information, an infrared light emitting diode (LED) was placed on the robot and a Nintendo Wii remote was attached overhead. The software uses the combination of the two to ascertain the $\mathrm{X}, \mathrm{Y}$ position of the robot. The software is interoperable with any gait or behavior learning algorithm.

\section{Example Gaits}

Evolutionary algorithms work best when they have a gradient to follow through a space rich with partial solutions. To get a sense of how randomly-generated gaits would perform, we chose a few gaits by setting random positions and having the robot interpolate between them in a repeated pattern. We found that many such patterns resulted in motion. Videos of several gaits are available on the website (Aracna, 2012).

\section{Conclusion}

Here we have introduced Aracna, a low-cost open source platform for evolutionary robotics. The complex kinematics along with the open source nature of the robot will provide an interesting and challenging platform for comparing walking gait algorithms. The updated platform is modular, allow- 
ing for low-cost replacement parts and varied leg designs. Future work can include modifying a single leg to have different linkage lengths, or to replace a kinematic joint with a compliant, or flexible, joint. Aracna will enable multiple users to compare data across a single lightweight, low-cost evolutionary robotic platform.

\section{Acknowledgements}

This work was supported by the National Science Foundation's Office of Emerging Frontiers in Research and Innovation (grant number 0735953) and an NSF Postdoctoral Research Fellowship in Biology to Jeff Clune (DBI-1003220).

\section{References}

Aracna (2012). Aracna open-source quadruped robot. Opensource hardware and software files are available at $\mathrm{http} / / /$ creativemachines.cornell.edu/aracna.

Bongard, J., Zykov, V., and Lipson, H. (2006). Resilient machines through continuous self-modeling. Science, 314(5802):11181121.

Clune, J., Beckmann, B., Ofria, C., and Pennock, R. (2009). Evolving coordinated quadruped gaits with the HyperNEAT generative encoding. In Proceedings of the IEEE Congress on Evolutionary Computation, pages 2764-2771.

Clune, J. and Lipson, H. (2011). Evolving three-dimensional objects with a generative encoding inspired by developmental biology. In Proceedings of the European Conference on Artificial Life, pages 144-148.

Clune, J., Stanley, K., Pennock, R., and Ofria, C. (2011). On the performance of indirect encoding across the continuum of regularity. IEEE Transactions on Evolutionary Computation, 15(4):346-367.

Clune, J., Yosinski, J., Doan, E., and Lipson, H. (2013). Automating the design of physical objects via interactive, crowdsourced evolution based on concepts from developmental biology. In preparation.

Gallagher, J., Beer, R., Espenschied, K., and Quinn, R. (1996). Application of evolved locomotion controllers to a hexapod robot. Robotics and Autonomous Systems, 19(1):95-103.

Gibson, I., Rosen, D., and Stucker, B. (2009). Additive manufacturing technologies: rapid prototyping to direct digital manufacturing. Springer Verlag.

Glette, K., Klaus, G., Zagal, J. C., and Torresen, J. (2012). Evolution of locomotion in a simulated quadruped robot and transferral to reality. In Proceedings of the Seventeenth International Symposium on Artificial Life and Robotics.

Hornby, G., Lipson, H., and Pollack, J. (2003). Generative representations for the automated design of modular physical robots. IEEE Transactions on Robotics and Automation, 19(4):703-719.

Hornby, G., Takamura, S., Yamamoto, T., and Fujita, M. (2005). Autonomous evolution of dynamic gaits with two quadruped robots. IEEE Transactions on Robotics, 21(3):402-410.
Kodjabachian, J. and Meyer, J. (1998). Evolution and development of neural controllers for locomotion, gradient-following, and obstacle-avoidance in artificial insects. IEEE Transactions on Neural Networks, 9(5):796-812.

Koos, S., Mouret, J., and Doncieux, S. (2011). The Transferability Approach: Crossing the Reality Gap in Evolutionary Robotics. IEEE Transactions on Evolutionary Computation, pages $1-25$.

Koza, J. (2003). Genetic programming IV: Routine humancompetitive machine intelligence. Kluwer.

Lipson, H., Bongard, J., Zykov, V., and Malone, E. (2006). Evolutionary robotics for legged machines: from simulation to physical reality. In Intelligent Autonomous Systems, volume 9 , page 9 .

Lipson, H. and Kurman, M. (2010). Factory@ home: The emerging economy of personal manufacturing. Papers in Science and Technology Policy.

Lipson, H. and Pollack, J. (2000). Automatic design and manufacture of robotic lifeforms. Nature, 406(6799):974-978.

Mondada, F., Bonani, M., Raemy, X., Pugh, J., Cianci, C., Klaptocz, A., Magnenat, S., Zufferey, J., Floreano, D., and Martinoli, A. (2009). The e-puck, a robot designed for education in engineering. In Proceedings of the 9th conference on autonomous robot systems and competitions, volume 1, pages $59-65$.

Nolfi, S. and Floreano, D. (2000). Evolutionary robotics: The biology, intelligence, and technology of self-organizing machines. MIT Press, Cambridge, MA.

Pfeifer, R., Bongard, J., and Grand, S. (2007). How the body shapes the way we think: a new view of intelligence. The MIT Press.

Sims, K. (1994). Evolving 3D morphology and behavior by competition. Artificial Life, 1(4):353-372.

Yosinski, J., Clune, J., Hidalgo, D., Nguyen, S., Zagal, J., and Lipson, H. (2011). Evolving robot gaits in hardware: the hyperneat generative encoding vs. parameter optimization. In Proceedings of the European Conference on Artificial Life, pages 890-897.

Zykov, V., Bongard, J., and Lipson, H. (2004). Evolving dynamic gaits on a physical robot. In Proceedings of Genetic and Evolutionary Computation Conference, Late Breaking Paper, GECCO, volume 4. 